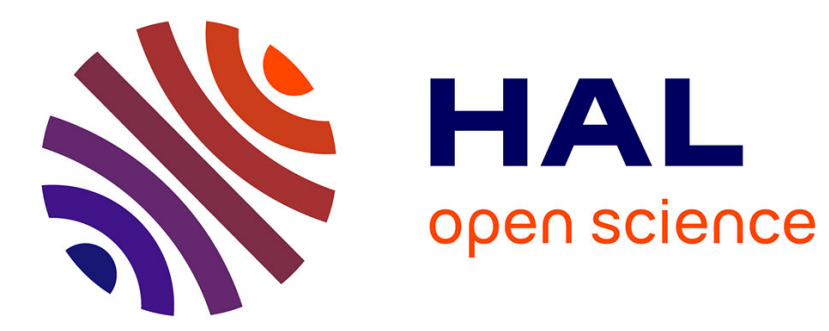

\title{
Regards sur l'enfant nouveau-né à Byzance Marie-Hélène Congourdeau
}

\section{- To cite this version:}

Marie-Hélène Congourdeau. Regards sur l'enfant nouveau-né à Byzance. Revue des études byzantines, 1993, Revue des études byzantines (51), pp.161-176. halshs-00682288

\section{HAL Id: halshs-00682288 \\ https://shs.hal.science/halshs-00682288}

Submitted on 24 Mar 2012

HAL is a multi-disciplinary open access archive for the deposit and dissemination of scientific research documents, whether they are published or not. The documents may come from teaching and research institutions in France or abroad, or from public or private research centers.
L'archive ouverte pluridisciplinaire HAL, est destinée au dépôt et à la diffusion de documents scientifiques de niveau recherche, publiés ou non, émanant des établissements d'enseignement et de recherche français ou étrangers, des laboratoires publics ou privés. 


\section{Regards sur l'enfant nouveau-né à Byzance}

In: Revue des études byzantines, tome 51, 1993. pp. 161-176.

\section{Résumé}

REB 511993 France p. 161-176

Marie-Hélène Congourdeau, Regards sur l'enfant nouveau-né à Byzance. — L'attitude envers le nouveau-né est un bon révélateur des éléments conscients et inconscients d'une culture. Dans la mentalité byzantine, elle exprime de manière privilégiée le conflit entre les composantes chrétiennes et non-chrétiennes. Une enquête dans des sources diverses (droit, droit canon, hagiographie, érotapokriseis, sources historiques et médicales ...) permet de dégager plusieurs attitudes : accueil et rejet (bréphotrophia, infanticide), protection et méfiance (l'enfant non baptisé, proie favorite des démons). Fragile, menacé, menaçant (la naissance multiple ou monstrueuse, funeste présage), proche du néant dont il vient, et où la plupart retournent rapidement, impur jusqu'à son baptême et cependant image de Dieu, le nouveau-né fascine, inquiète, émeut. II offre aussi à l'Église byzantine l'occasion d'affirmer la spécificité de son anthropologie et de façonner une pratique différente de celle des civilisations antiques.

Citer ce document / Cite this document :

Congourdeau Marie-Hélène. Regards sur l'enfant nouveau-né à Byzance. In: Revue des études byzantines, tome 51, 1993. pp. 161-176.

http://www.persee.fr/web/revues/home/prescript/article/rebyz_0766-5598_1993_num_51_1_1875 


\section{REGARDS \\ SUR L'ENFANT NOUVEAU-NÉ À BYZANCE *}

\section{Marie-Hélène CONGOURDEAU}

L'attitude envers le nouveau-né dévoile les éléments inconscients d'une culture. Dans la mentalité byzantine, elle exprime le conflit entre les composantes chrétiennes et non chrétiennes. Fragile, menacé, menaçant, proche du néant dont il vient et où la plupart retournent rapidement, impur jusqu'à son baptême et cependant image de Dieu, le nouveau-né fascine, inquiète, émeut. Il offre aussi à l'Église byzantine l'occasion d'affirmer son anthropologie spécifique et de façonner une pratique différente.

\section{I)ES RÉalités TRAgiQUEMENT têtUES}

La naissance fut longtemps une épreuve à haut risque. Les Byzantins durent affronter la tragique réalité : mortalité des bébés et des femmes en couches, enfants malformés ou non viables, rejet de l'enfant importun.

\section{A) Quand la vie à la mort se marie}

Le nouveau-né est un être fragile dont la vie toute neuve risque à tout moment de lui être arrachée. De nombreux textes, d'histoire ou de fiction, témoignent de cette peur: Michel Psellos rapporte qu' «autrefois, les rois de Perse ne voyaient pas tout de suite les enfants nouveau-nés, et ils ne prenaient pas tout de suite dans leurs bras les fruits de leurs entrailles (parce qu')ils craignaient pour les nourrissons, qu'ils trouvaient trop fragiles, (...) que la mort ne les

* Cet article est la version développée d'une communication faite au XVII" Congrès international des Ftudes byzantines (l niversité $\mathbf{M}$. V. Lomonosov. Moscou, 8-15) aoùt 1991).

Revue des Études Byzantines 51, 1993, p. 161-176. 
enlève" ${ }^{1}$. L'empereur Andronic II, ayant perdu trois enfants avant leur naissance, craignait pour la vie de sa fille nouveau-née et recourut, pour sa sauvegarde, à un rituel recommandé par une matrone "pour la survie des nouveau-nés" ${ }^{2}$.

L'accouchement difficile est un topos de la littérature hagiographique, et l'enfant mort-né, non viable (alrophos) ou mort prématurément (aôros), occupe tous les étages de la pensée byzantine, des traités sur la providence divine ${ }^{3}$ aux recueils d'horoscopes ${ }^{4}$ en passant par les Vies de saints ${ }^{5}$, sans parler des terreurs inspirées par les divers visages de la démone Gillô, tueuse de nouveau-nés ${ }^{6}$.

La mort de la mère en couches n'est pas moins redoutée. Tel fut le sort de la sœur de Psellos ?. Et les textes hagiographiques abondent en récits d'accouchements pathologiques où la vie de la mère et de l'enfant sont en jeu : parfois la mère du saint meurt ${ }^{8}$ ou manque de

1. Michel Psellos, l ettre 157 à l'épi tôn kriséôn, èd. Sathas, MB, V, p. 409; trad. fr. A. Leroy-Molinghen, La descendance adoptive de Psellos, Byz., 39, 1969, p. 302.

2. Pachymère, II, III, 32, Bonn II, p. 276-277: on dresse les icônes des douze apôtres, avec douze cierges allumés d'égale longueur, et l'on psalmodie jusqu'à extinction des cierges; l'enfant reçoit le nom de l'apotre dont le cierge s'éteint en dernier. C'est ainsi que la fille d'Andronic fut prénommée Simonis, du nom de l'apôtre SimonPierre.

3. Cf. Diodore de Tarse, Fragment 69 sur l'Octateuque, èd. J. Deconninck, p. 143 ; cf. aussi $\mathrm{J}$. Devreesse, Les anciens commenlateurs grecs de l'Octateuque et des Rois (Fragments tirés des chaines), Vatican 1959, p. 162. - Anastase le Sindïte, Erotapokriseis, qu. 88, $P G$ 89, 716. - Michel Glykas, A poriai, c. 37, éd. Eustratiadès, I, p. 422 s.

4. Cf. G. Dagron, Le fils de Léon Ier, témoignages concordants de l'hagiographie et de l'astrologie, An. Boll. 100, 1980, p. 273-274. Les manuels d'astrologie évoquent les questions qui peuvent être posées aux astrologues. Plusieurs de ces questions concernent la viabilité du nouveau-né : cf. par exemple Paris. Supp. Gr. 1148 (16 s.), f. $166-170$; Laur. Plut. 28,33 (16 s.), f. 57 : "savoir si le nouveau-né ne vivra pas" $(=C C A(i, I, \operatorname{cod} .11)$.

5. La Vie de Mélanie (éd. D. Gorce, SC 90) comporte les deux drames : le second enfant de Mélanie, né prématurément - aóros - ne survit pas à son baptème (c. 5); Mélanie, par ses prières, obtient l'expulsion d'un enfant mort du sein de sa mère (c. 61).

6. Cf. la monographie d'Irène Sorlin, Striges et Géloudes. Histoire d'une croyance et d'une tradition, $T M 11,1991$, p. 411-436. On trouve aussi mention d'esprits mauvais qui tuent les enfants en bas àge dans la Vie de saint Syméon Stylite le Jeune, éd. Van den Ven, 1962, par. 139 et 231. A propos de la démone Gillo, ef. également le cod. Athen. Bibl. Soc. Ilist. 210 du 18" s. (=CCAG, X, cod. 25), f. 64" : Phylactère pour les petits enfants, à propos de Gelo", et f. $86^{\mathrm{v}}$ : "Phylactère contre l'impure Gelo", etc.

7. Psellos, Eloge funèbre de sa mère, èd. Sathas, V, p. 27-28.

8. Cf. le Marlyre de Théodore Tiron par Vicéphore Ouranos, ed. F. Ilalkin, An. Boll. 80,1962, p. 314 . 
mourir ${ }^{9}$ en couches. Le plus souvent, le saint opère un miracle en provoquant l'heureuse issue d'un accouchement long et périlleux ${ }^{10}$.

Significatif est le cas de l'enfant qui entraîne sa mère dans la mort : ainsi périt l'impératrice Eudoxie, en tentant d'expulser par la magie le petit corps qui se décomposait en elle depuis quatre jours ${ }^{11}$.

Le nouveau-né est alors non seulement menacé mais menaçant, puisqu'il peut entraîner sa mère avec lui dans la mort. Or la vie de la mère est toujours prioritaire, comme en témoigne le recours des médecins à l'embryotomie dans les cas désespérés ${ }^{\mathbf{1 2}}$.

\section{B) Quand l'enfant est difforme}

Lorsque l'enfant naît vivant mais mal formé, la pratique constante dans l'Antiquité est de ne pas le laisser vivre. De Sparte à Rome en passant par la République de Platon, on étouffe, noie ou expose sur les chemins les enfants non conformes. A côté de cette "euthanasie" active (pour employer un euphémisme anachronique), une "euthanasie" passive est la règle générale : le médecin Soranos d'Éphèse, prince des accoucheurs, l'expose sereinement dans son manuel destiné aux sages-femmes : "(La puériculture) recherche quels sont ceux des nouveau-nés qui valent la peine qu'on les élève" ${ }^{13}$. La sage-femme doit "se rendre compte si l'enfant vaut la peine qu'on l'élève", c'est-à-dire qu'on le nourrisse (anatrophè) ${ }^{14}$. Avec le temps cependant, les médecins cherchent à maintenir en vie des enfants atteints de malformations légères ou curables ${ }^{15}$.

9. If. Theodoret de Cyr. Histoire Philothée IX, 14. Il sagit de la propre mère de Théodoret. (if. également la Vie de Théophano de Thessalonique, éd. E. Kurtz. Zwei griechische Texte über die III. Theophano, die Gemahlin Kaiser Leo VI. p. 2.

10. Vie d'Ignace de Constantinople par Nicétas de Paphlagonie. P(; 105, 488 s.: Vie de Porphyre de Gaza par Marc le Diacre, c. 28, éd. H. Grégoire et M.-A. Kugener, Paris 1930 : ces deux saints évitent, par leur intervention. l'embryotomie projetée par les médecins. Vie de Marcel l'.1 cémète, c. 21. éd. G. Dagron, An. Boll. 86. 1968, p. 287-321: lie de Syméon Salos par Léontios de Véapolis : exceptionnellement. laccouchement laborieux est presente ici comme un chatiment.

11. Kèdrènos. Historiarum Compendium I, 585 s.: Vie anonyme de Jean Chrysostome, An. Boll. 94, 1976, p. 353 : les fragments de ces deux sources qui relatent la mort de l'impératrice Eudoxie sont analysés par W. Fink. Geburtshilfe in Byzanz.'. Zwei Beispiele aus dem frühen 5. Jahrhundert. .JÖB 36. 1986. p. 333-37.

12. Pour l'embryotomie, out re les textes cités note 9. cf. Celse. VII. 29, 4: on soulignera que la description trés détaillée de cette opération ne concerne, chez ce médecin. que l'extraction dun foetus mort dans le sein: Soranos. IV. 3, 1 : Tertullien, Ie an. 25. 5: Macaire. Hom. 43. P(; 34, 776.

13. Soranos, Des maladies des femmes 11, 4, ed. D. Gourevitch, 1990, 11. p. 16.

14. Idem, II. 5: 1). (iourevitch. II, p. 16-17. La note 99 (p. 85) se rapportant à ce passage énumère, d'après la littérature médicale antique. les cas ou le nouveau-ne ne sera pas nourri.

li). Ibidem, nolde ys 


\section{C) L'enfanl nié}

L'enfant normal lui-même n'est pas toujours le bienvenu. L'infanticide est largement pratiqué dans toute l'Antiquité, au point que Grégoire de Nysse, traitant de la mort des jeunes enfants, énumère tranquillement les trois principales causes de mortalité infantile : le nouveau-né meurt "soit exposé, soit étouffé (ou noyé), soit enlevé naturellement par une maladie ${ }^{16}$.

Si l'on en croit la législation impériale ou canonique d'époque byzantine, les principales formes d'infanticide sont l'abandon (ou l'exposition), la négligence volontaire, le refus de mettre l'enfant au sein ${ }^{17}$. Mème en tenant compte de l'inertie et du conservatisme des textes législatifs, on ne peut nier la permanence de ces pratiques très tardivement.

\section{II. Élé.Ments pour une nouvelle anthropologie}

En se généralisant dans l'empire romain, le christianisme apporte une nouvelle vision de l'être humain, qui devrait entraîner de nouvelles pratiques.

L'anthropologie chrétienne est tributaire des anthropologies antiques; comme elles, elle voit la spécificité de l'homme dans sa raison, d'où le statut particulier de l'enfant qui n'a pas encore atteint l'"âge de raison»: Pour Grégoire de Nysse, le nouveau-né n'est pas encore un "homme" à strictement parler, car il n'est pas logikos ${ }^{18}$. Cependant, il est déjà respectable, car il porte l'image de Dieu.

La création à l'image de Dieu, héritée du judaissme (qui en tire les mêmes conséquences morales de respect de la vie humaine, même enfantine), est le fondement de la nouvelle anthropologie élaborée par

16. Grégoire de Nysse, De infantibus praemature abreplis, $P G$ 46, $168 \mathrm{~B}$. Cf. Idem, De anima et resurrectione, $P G$ 46, 137 : “... à propos des nouveau-nés qu'on expose ou qu'on étouffe, ou de ceux qui meurent de mort naturelle ...».

17. Le Code justinien, dans un article qui sera repris par toute la législation postérieure, indique : "Est meurtrier non seulement celui qui étouffe (ou étrangle, ou noie : praefocat, pnigô) le nouveau-né, mais aussi celui qui l'abandonne (ou le jette : abicit, rhiptô), qui lui refuse la nourriture, et celui qui l'expose dans des lieux publics à la charité d'autrui, charité qu'il n'a pas eue lui-même" (Dig. 25, 3,4). On retrouve ces diverses formes d'infanticide dans la législation canonique et les pénitentiels, y compris le refus de nourriture, qui représente dans la littérature médicale la forme habituelle d'"euthanasie». Cf. Jean le Jeûneur, Logos pros ton mellonta .., PG 88, 1924 A : "Sur celles qui empêchent de téter leurs propres enfants".

18. Grégoire de Nysse, De infantibus .., $P G 46,168 \mathrm{~B}$. C.f. à l'inverse Némésios d'Émèse, Sur la nalure de l'homme, c. 2 in fine, $P G 40,584$. 
les Pères de l’Église. S'y ajoute le précepte : "Tu ne tueras pas" du Décalogue ${ }^{19}$.

Mais c'est surtout I'Incarnation du Fils de Dieu qui appose le sceau du divin sur l'humanité : en se faisant homme dans le sein de Marie, c'est-à-dire en passant par toutes les étapes de l'embryon, du nouveau-né, de l'enfant, etc., le Christ a sanctifié tous les âges de la vie : tout nouveau-né est l'image de l'enfant Jésus dans la mangeoire de Bethléem ${ }^{20}$.

Hnfin, tout ètre né vivant est appelé à recevoir le salut par le baptème. Ce qui renforce l'obligation de préserver la vie du nouveau-né : il est inconcevable de priver un petit d'homme de la vie éternelle.

Tous ces éléments vont progressivement changer le regard porté sur le nouveau-né, mais au prix d'une longue patience, car en ce domaine crucial, les irınovations apportées par le christianisme bouleversent des mentalités et des pratiques millénaires, alors que les réalités (mortalité, maladies, naissance importune) n'ont pas changé.

\section{L NE MENTALITÉ NOLVELLE EN GESTATION}

\section{A) Des tabous christianisés}

I a naissance est le domaine des tabous. Bien souvent, le christianisme ne fait pas autre chose que d'habiller des croyances préchrétiennes.

\section{I.a naissance est impure}

Un tabou tenace est l'impureté de la naissance. Le mystère de cet être venu du néant, le sang et les déjections qui accompagnent sa sortie ont dès longtemps accrédité l'idée d'une souillure de la naissance $^{21}$.

a) Impureté de la femme en couches

Pour les Grecs, tout ce qui touche l'accouchement était impur; ils avaient élaboré un rituel d'intégration du nouveau-né, de réintégra-

19. Frode $20,13$.

20. Ce thème, qui court tout le long de la tradition patristique, est déjà dans Irénée. Conire les hérésies II, 22, 4 (èd. A. Rousseau, SC 293-294) : “... Il a sanctifié tous les àges par la ressemblance que nous avons avec lui. C'est en effet tous les hommes ... qui par lui renaissent en Dieu : nouveau-nés, enfants, adolescents, jeunes hommes, hommes d'âge. C'est pourquoi il est passé par tous les âges de la vie : en se faisant nouveau-né, il a sanclifié les nouveau-nés: en se faisant enfant, etc.".

21. Sur la notion de souillure et d'impureté de la naissance dans la mentalité grecque, et ses prolongements à l'époque byzantine, cf. Irène Sorlin, art. cit., note 5 , p. 432-434. I a novelle 17 de Léon VI avance une explication médicale à l'impureté du sang menstruel et des lochies : c'est qu'il s'agit, en bonne physiologie aristotèlicienne. de résidu et donc d un sang superflu. 
tion de la mère dans la communauté et de purification de la maison natale et de quiconque avait touché le nouveau-né ou sa mère.

En même temps que l'héritage antique, les Byzantins ont recueilli celui du judaïsme. Le Lévitique prévoit quarante jours d'éviction de la femme qui a accouché d'un garçon, plus de quatre-vingts pour une fille ${ }^{22}$. Le rite de la purification, que l'on retrouve dans la fête de la Purification de Marie le 2 février (quarante jours après Noël), clôt cette période.

Le christianisme a intégré ce double héritage, en lui donnant un visage particulier.

\section{* Les lochies de Marie}

Les canons ont conservé le souvenir d'une coutume qui remonte aux temps pré-chrétiens : les épilochia (terme absent des dictionnaires classiques) célèbrent les lochies de la femme par le partage d'un gâteau de froment. Or certains Byzantins s'étaient avisés de célébrer les épilochia de Marie le lendemain de Noël. Le canon $79 \mathrm{du} 6^{\mathrm{e}}$ concile interdit cette coutume, qui heurte la christologie orthodoxe en laissant croire que l'accouchement de Marie fut un accouchement ordinaire ${ }^{23}$. Les commentaires de ce canon soulignent le lien entre douleur de l'accouchement et lochies d'une part, conception sans semence et accouchement sans lochies d'autre part. Marie n'a pas de lochies parce qu'elle a conçu sans semence; sperme et sang partagent la même impureté ${ }^{24}$.

22. L'impureté du sang dans la tradition biblique n'est pas morale, mais rituelle : la perte de sang au moment de l'accouchement est impure parce que le sang est sacré, il contient l'àme qui appartient à Dieu. Cf. E. Lepicard, L'embryon dans la Bible et la tradition rabbinique, Ethique. La vie en question 3 (Hiver 1992), p. 38, note 3.

23. Sixième concile (in Trullo), canon 79 (RP II, p. 442-443) : "Confessant que le divin enfantement de Marie fut sans lochies (alocheuton), comme il fut sans semence (asporôs), et le proclamant à tout le troupeau, nous incitons à se corriger ceux qui par ignorance font ce qui ne convient pas. C'est pourquoi, puisque certains, après le jour de la nativité sainte du Christ notre Dieu, font cuire de la farine de froment et la partagent entre eux, sous prétexte d'honorer les lochies de la Vierge Mère sans tache, nous prescrivons que les fidèles ne fassent rien de semblable. Car ce n'est pas honorer la Vierge, qui a enfanté dans la chair le Verbe incirconscriptible d'une façon qui dépasse l'esprit et la raison, que de prescrire et de souscrire que ce qui concerne son indicible enfantement s'est passé comme à l'ordinaire et comme pour nous. Si donc quelqu'un à partir d'aujourd'hui est surpris en train d'agir ainsi, si c'est un clerc, qu'il soit déposé ; si c'est un laïc, qu'il soit excommunié». Sur la Vierge ayant accouché sans douleur et sans souillure, cf. Grégoire de Nysse, Homélie XIII sur le Cantique des Cantiques, éd. Jaeger, VI, p. $387-388$.

24. La Bible unit dans le même tabou l'accouchement (avec les lochies) et l'écoulement de sperme : Lévilique 12 et 15 . Cf. les commentaires du canon 79 du sixième concile par Zonaras, Balsamon et Blastarès : RP II, p. $443 \mathrm{~s}$. 
* I a accouchée impure

Le canon 2 de Denys d'Alexandrie interdit aux femmes de venir à l'église et de participer aux mystères pendant leurs règles. Les commentateurs de ce canon évoquent la même interdiction pour les femmes durant quarante jours après un accouchement: ce qui confirme que c'est bien le sang des lochies qui rend l'accouchée impure. Des exceptions sont admises en cas de péril de mort : le simple bon sens suffisait pour montrer que ces femmes étaient plus exposées que d'autres ${ }^{25}$.

b) Le nouveau-né impur

Limpureté de la femme se communique à ceux qui l'approchent. Pierre le chartophylax, au $11^{\mathrm{e}} \mathrm{s}$., se voit poser la question suivante : "Si une femme accouche, et si certains entrent dans sa maison, quelle pénitence doivent-ils subir pour pouvoir communier?". A quoi il répond qu'à condition de s'être lavés, ils ne peuvent être exclus de la communion (on retrouve la notion d'une souillure qui nécessite une purification) ${ }^{26}$.

Mais c'est par excellence le nouveau-né qui partage l'impureté de sa mère : il vient de son sang, et il est un mélange de vie et de non-vie. Les croyances populaires brodent sur ce thème : le corps du nouveau-né est mis en parallèle avec le cadavre (avec qui il partage le port de bandelettes) ${ }^{27}$; de mêrne, pendant quarante jours (temps qui s'écoule entre la naissance et la purification de la mère), la démone Gillo menace sa vie ${ }^{28}$.

Un amalgame s'opère entre la souillure de la naissance ct la souillure du péché originel. En occident, avec la controverse antipélagienne d'Augustin, se développe la doctrine de la tache originelle transmise par la génération. Mais à Byzance aussi, les canonistes affirment que même les nouveau-nés sont baptisés "pour la rémission des péchés", et que par conséquent ils sont souillés par le péché avant mème de l'avoir commis ${ }^{29}$. De cette souillure, le baptême les purifie.

25. Canon 2 de Denys d'Alexandrie et ses commentaires (RP IV, p. 7-9); cf. leon VI. Novelle 17.

26. Pierre Chartophylax, RP V. p. 372.

27. Cf. G. Dagron, Troisième, neuvième et quarantième jour dans la tradition byzantine : temps chrétien et anthropologie, in Le temps chrétien de la fin de l'Antiquité au Moyen age, $H^{e}-\mathrm{X} H^{e}$ s., Paris 1984, p. 419-430.

28 . (if. I. Sorlin, art. cit., note 6 .

29. Canon 110 de Carthagène et ses commentaires (RP II I, p. 561 s.). Blastarès (RP, p. 117) affirme que c'est l'impureté du processus de génèration sexuelle qui rend nécessaire pour lenfant la purification du baptème. 
* Quand faut-il baptiser?

En bonne logique, c'est donc le quarantième jour, quand la mère est purifiée, que le nouveau-né reçoit lui aussi la purification du baptême. Deux faits contribuent à raccourcir ce délai.

Le premier est la nécessité d'une cohérence théologique. Si c'est le baptême qui purifie de la souillure, point n'est besoin d'attendre que cette souillure ait physiquement cessé (arrêt des lochies) pour l'administrer.

Le second est le danger mortel couru par beaucoup de nouveau-nés. Il y aurait de l'inconscience à les laisser mourir sans avoir reçu le baptême. C'est pourquoi des dérogations sont admises par les plus rigoristes : si le nouveau-né est "mal venu», on peut le baptiser dès le huitième jour, voire dès la naissance ${ }^{30}$.

* Sort des enfants morts sans baptême

Il importe en effet de ne pas laisser un petit d'homme mourir sans recevoir la régénération. Quel grave danger courait-il donc? Le sort des enfants morts sans baptême a préoccupé la conscience chrétienne au long des siècles. Mais c'est surtout en occident que la controverse, sous l'influence d'Augustin, a donné lieu à toute une réflexion. L'orient chrétien semble plus pragmatique : le bons sens y tient sur ce point autant de place que la théologie. Déjà Grégoire de Nazianze, qui recommandait le baptême précoce, estime que celui qui meurt non baptisé, mais sans qu'il y ait de sa faute (c'est le cas des nouveau-nés), ne saurait recevoir dans l'autre monde ni récompense ni châtiment ${ }^{31}$. Anastase le Sinaïte répond elliptiquement à qui l'interroge sur le sujet : puisque Dieu a dit que la faute des pères ne saurait retomber sur les enfants, la négligence des parents ne doit pas mener dans la géhenne les petits êtres morts sans baptême; nous n'aurons pas plus de précisions : il est des questions qui n'ont pas de réponse sur cette terre ${ }^{32}$.

30. Cf. canon 38 de Vicéphore le Patriarche ( $\left(9^{e} \mathrm{~s}\right.$.) : baptême possible dès trois jours; novelle 17 de Léon VI (10 s.) : dès avant le huitième jour; Nicolas III Grammatikos (Réponses à l'évêque de Zètounion, $12^{*}$ s., èd. Darrouzès, Kathègètria. Essays presented to Joan Hussey, Camberley 1988, p. 327-343) : la coutume est de baptiser au quarantième jour, mais on peut le faire dès la naissance en cas de nécessité, aussitôt après l'avoir lavé (toujours la nécessité de la purification : l'enfant doit être débarrassé du sang de sa mère).

31. Grégoire de Nazianze, disc. 40, 23, $P G 36,389$. Il semble que ce soit aussi la position du Ps. Justin des "Réponses aux orthodoxes" (Q. 56, PG 6, 1927).

32. Anastase le Sinaite, Érolap. qu. 81, PG 89. 
Le même Anastase, dans ses Récils édifianls, est à l'origine d'un très petit corpus de textes concernant la résurrection d'un enfant en vue du baptême (alors que ce thème fleurit considérablement en occident, signe d'une plus grande angoisse à ce sujet ${ }^{33}$ ): un homme vient de nuit quérir un prêtre pour lui demander de venir baptiser son enfant en péril de mort. Le prêtre se hâte, mais arrive trop tard : l'enfant meurt durant les préparatifs du baptême. Le prêtre, en vertu de son pouvoir sacerdotal, supplie Dieu de redonner son âme à l'enfant le temps de le baptiser, car il n'y a pas eu négligence; l'enfant ressuscite, reçoit le baptême et s'endort à nouveau dans la mort ${ }^{34}$. Ce récit est repris par Michel Glykas ${ }^{35}$, puis de nouveau par Théognoste, qui l'insère dans son chapitre sur le sort des enfants morts sans baptême (où il reprend la doctrine d'Anastase). Ce dernier ajoute une finale plus intimiste : le prêtre embrasse l'enfant baptisé et lui dit : "Va ton chemin, mon enfant, vers le royaume des cieux" ${ }^{36}$.

* Quand l'enfant est purifié avant sa mère

Un problème ne pouvait manquer de se poser à l'esprit incisif des Byzantins : que faire lorsque l'enfant, en péril de mort, a été baptisé avant le terme de la purification de sa mère? Autrement dit, quel peut être le rapport entre un enfant purifié par le baptême et une mère impure? Il se trouva des canonistes pour estimer qu'alors la mère ne pouvait plus toucher son nourrisson, ni donc l'allaiter, et qu'il fallait trouver une nourrice baptisée pour allaiter le petit chrétien : tel Nicéphore le patriarche, au $9^{e} \mathrm{~s} .{ }^{37}$. Fort heureusement, certains firent prévaloir le bon sens et la charité sur la rigueur : Pierre le chartophylax, au $12^{\mathrm{e}} \mathrm{s}$., répond sèchement à ceux qui lui posent la question : "Qu'elle allaite son bébé, afin qu'il ne meure pas!" ${ }^{38}$.

2. Le nouveau-né non baptisé, proie des démons

Ainsi, le nouveau-né non baptisé a un statut ambigu : image de Dieu, image du Christ de Bethléem, mais non purifié de la souillure originelle. Dans sa double condition innocente et souillée, il est le lieu de tous les fantasmes.

33. C.f. les travaux de J. Gélis sur les «sanctuaires à répit», en particulier L'arbre et le fruit. Paris 1984, p. $509-510$.

34. Cf. F. Nau, Ie texte grec des récits utiles à l'âme d'Anastase (le Sinaïte), Oriens Christianus :3, 1903 , p. 82.

35. Michel Glykas. Aporiai. 61 ("Que le lien imposé par le prêtre ne peut être dénoué»), II, éd. Eustratiadès. Alexandrie 1912, p. 146.

36. Théognoste. Thesauros, XV, 4, éd. J. Munitiz, p. 129 s.

37. Nicephore te Patriarche. canon 38. RP IV. p. 131.

38 . Nierre Chartophylax. RP V. p. 372. 
a) Pouvoirs anormaux du nouveau-né

En occident, le thème du nouveau-né aux pouvoirs anormaux est illustré principalement par la figure de Merlin. Or Merlin est l'enfant du démon. Ce qui jette un éclairage significatif sur les récits parallèles de l'hagiographie byzantine: le nouveau-né qui disculpe sa mère accusée d'adultère, ou qui dénonce son père ${ }^{39}$. Le nouveau-né a un rapport privilégié, mais ambigu, au monde invisible.

b) Pratiques magiques impliquant des nouveau-nés

Cette ambiguïté est, dans toutes les civilisations, à l'origine de pratiques magiques qui utilisent le nouveau-né et ce qui le touche. Michel Glykas rapporte que, l'empereur Constantin se trouvant atteint de la lèpre, les prêtres de Jupiter Capitolin lui conseillèrent de s'asperger du sang de nouveau-nés sacrifiés ${ }^{40}$. D'après un apocryphe de Psellos, les manichéens engrossent des filles puis tuent l'enfant à sa naissance et boivent son sang pour recevoir en eux les démons ${ }^{41}$. Nous sommes ici chez les païens (prêtres de Jupiter) ou les hérétiques (manichéens). Mais Balsamon nous rapporte, dans son commentaire des canons du $6^{\text {e }}$ concile, l'histoire d'un clerc surpris en possession d'une coiffe de nouveau-né dont il comptait faire un usage magique ${ }^{42}$. Et c'est bien en pays byzantin que, selon Nicéphore le Patriarche, les habitants de Pergame, en 717, firent bouillir un nouveau-né pour obtenir la "potion magique" qui leur permettrait de repousser les Arabes ${ }^{43}$.

c) Gillo la tueuse de nouveau-nés

Nous ne reprendrons pas ici ce qui a été fort bien exposé par Irène Sorlin à propos de la démone Gillo et de ses avatars païens et chrétiens. Soulignons seulement que le nouveau-né non baptisé est la proie spécifique de la démone dans la plupart des versions chrétiennes, le délai de huit ou de quarante jours laissé à l'action de Gillo correspondant aux dates du baptême ${ }^{44}$. Toute la conception du baptême (à commencer par la présence d'un ange gardien) s'oppose d'ailleurs à la possibilité d'un tel danger pour un enfant baptisé.

39. Cf. P. Canart, Le nouveau-né qui dénonce son père, An. Boll. 84, 1966, p. $309 \mathrm{~s}$.

40. Michel Glykas, Annales IV, Bonn p. 460. 141.

41. Pseudo-Psellos, Péri énergeias daimonôn, éd. P. Gautier, REB 38, 1980, p. 140-

42. RP II, p. 446.

43. Nicéphore le Patriarche, Breviarium, 53, $P G 100,957$.

44. Irène Sorlin, art. cit., note 6 . 
d) Démons de l'accouchement et bébés possédés

Le même traité du Pseudo-Psellos sur les démons, reprenant probablement un passage du Testament de Salomon, rapporte la croyance populaire en un démon qui "apparaît sous un aspect féminin à toutes les femmes en couches". L'auteur ajoute un peu plus loin : "Aucune difficulté que le type de démon qui assaille les femmes en couches soit vu sous une apparence féminine, puisqu'il est lascif et qu'il apprécie les liquides malpropres" ${ }^{45}$ : preuve du dégoût que provoquent l'accouchement et tout ce qui relève du monde féminin ${ }^{46}$.

3. Monstres et prodiges

La naissance d'un enfant difforme défie la pensée. Dans les cas extrêmes, l'interrogation première est de savoir s'il s'agit d'un être humain.

Un manuscrit astrologique propose des recettes "pour savoir si ce qui est né est un homme ou autre chose" ${ }^{47}$ et "pour savoir s'il s'agit de la naissance d'un homme ou d'un monstre" ${ }^{48}$.

L'Épitomè, reprenant le Digeste, donne à l'être difforme une existence juridique : "Si une femme accouche d'un monstre ou d'un prodige n'ayant pas forme humaine, il n'est pas considéré comme un enfant». Suit la définition du monstre (qui combine la forme humaine avec celle d'un animal) et du prodige (qui n'a rien d'humain). En revanche, "celui qui naît avec six doigts est compté au nombre des enfants de son père "49.

Comme tout ce qui angoisse, le monstre fascine. Les chroniqueurs ont recueilli avec soin les mentions d'êtres hors-normes : de la naissance de quadruplés (qui d'ailleurs ne vivent pas) ${ }^{50}$ à la venue à Constantinople de Siamois venus d'Arménie ${ }^{51}$, en passant par la nais-

45. Pseudo-Psellos, Péri énergeias daimonôn, éd. P. Gautier, REB 38, 1980, p. 164170 (c'est nous qui soulignons). Cette affirmation est précédée du récit de la délivrance d'une femme possédée pendant son accouchement. Le démon s'exprime par la bouche de cette femme dans la langue des Arméniens (race considérée comme impure par les Byzantins). Cf. Testament de Salomon, $P G$ 122, 1334 s. : le démon qui menace les femmes en couches est Obizouth.

46. Cf. aussi le miracle accompli par Posidonios de Thèbes, qui expulse un démon empèchant une femme possédèe d'accoucher: Histoire lausiaque, c. 36.

47. CCAG, I, cod. 11 (Plut. 28, cod. 33), f. 55'.

48. Ibidem, f. 203 .

49. Digeste 14; Épitomè IV, 12.

50. Thèophane, Chronographie, CSHB, p. 106-107. L'événement eut lieu à Antioche vers l'an 375. L'Épitomè relate de mème la naissance à Alexandrie de quadruplés, suivis quarante jours plus tard d'un cinquième frère: IV, 11 ; cf. Digeste $5,4,3 ; 46,3,36$.

51. Théophane Continué. CSHB, p. 133 ; Léon Grammatikos, p. 326; Michel Glykas. p. 560. Cf. une analyse détaillée de l'événement (qui met en scène deux Arméniens 
sance d'un enfant sans yeux ni mains, et pourvu d'une queue de poisson : incident mis en parallèle avec la naissance, hors du territoire byzantin, d'un enfant à quatre pieds et d'un autre à deux têtes ${ }^{52}$.

Le "monstre" à queue de poisson (selon la définition légale) est mis à mort aussitôt, sa naissance étant assimilée à un funeste présage; de même les Siamois, après avoir été un spectacle de cirque, sont exilés de Constantinople. Et Eustathe de Thessalonique rapporte le cas de cette femme du Péloponnèse qui, ayant mis au monde neuf enfants en une seule fois, en jette sept dans le fleuve ${ }^{53}$.

Funestes présages dès l'Antiquité, les monstres et prodiges sont souvent considérés, dans les milieux chrétiens, comme des châtiments de Dieu pour une faute commise par les parents, comme l'union conjugale durant le temps impur des règles de la femme ${ }^{54}$.

\section{B) Émergence de nouvelles pratiques}

1. Le combat de la rationalité

La christianisation de l'empire ne se traduit pas seulement par l'adaptation des vieux tabous à l'imaginaire chrétien. Contre le paganisme et ses coutumes, les Pères ont tenté d'établir de nouveaux modes de pensée, et la première tâche fut souvent de faire prévaloir la raison sur la superstition.

Dans la lignée des médecins et philosophes antiques qui avaient lutté contre la conception magique du monde ${ }^{55}$, bien des auteurs byzantins interprètęnt les tabous des morts prématurées et des naissances monstrueuses dans une perspective "naturaliste».

réunis par le nombril) dans (F. F. Pentagalos et J. G. Lascaratos, Bull. of Hist. of Medic. 58, 1984.

52. Théophylacte Simokatès, VI, 1 (= Photios, Bibliothèque, cod. 65) le situe sous le règne de l'empereur perse Chosroès. Léon Grammatikos (p. 139) et Michel Glykas (Annales, p. 507) le datent du règne de Maurice Tibère. C'est chez ces deux derniers chroniqueurs que l'on trouve le parallèle avec d'autres naissances monstrueuses. Dans la Vie de Syméon Stylite le Jeune, une femme met au monde un enfant aveugle sans marque des yeux; les parents vont voir le saint pour lui demander pourquoi il est né ainsi (il va sans dire que Syméon lui redonne des yeux) : Vie de Syméon Stylite le Jeune, éd. Van den Ven, 1962, par. 117.

53. Eustathe de Thessalonique, Parekb. eis tèn Omèr. Iliad., éd. M. van der Valk, III, Büll 1979, p. 475-6.

54. Dans le miracle 15 de Cyr et Jean, l'auteur attribue la lèpre d'un malade à l'union de ses parents durant le temps des règles : $P G 87,3468$. Cette opinion est très ancienne et très répandue : cf. Théodoret de Cyr, Qu. 14 sur le Lévitique; Jean le Jeûneur, Kanonikon (Rhallès-Potlès IV, p. 441); Léon VI, Novelle 17. Sur ce thème, cf. S. V. Troianos, L'embryon dans le droit canon byzantin, Elhique. La vie en question 4 (Printemps 1992), p. 55-57.

55. C. principalement Hippocrate, La maladie sacrée (éd. en cours aux Belles Lettres par J. Jouanna). 
Diodore de Tarse, polémiquant contre les tenants du Destin, explique l'existence des monstres (êtres qui ont des éléments en trop, par exemple plusieurs têtes) et des êtres mutilés par une déviation de la nature vers l'excès ou le manque ${ }^{56}$.

Le pseudo-Athanase, dans son homélie sur l'aveugle-né, explique les anomalies par un défaut de la semence masculine ou de la matrice ${ }^{57}$.

Le débat sur la Providence est un lieu privilégié pour cette recherche d'une cohérence logique autant que théologique.

Pour Anastase le Sinaite au $7^{\mathrm{e}} \mathrm{s}$., si la mort des nouveau-nés demeure scandaleuse ${ }^{58}$, elle ne saurait remettre en cause la providence. Dans sa question 127 , il rapporte le cas d'une femme qui avait vu mourir ses trois premiers nourrissons; à la naissance du quatrième, I)ieu lui inspire de ne pas le nourrir mais d'avoir recours à une nourrice; il s'agissait en effet non d'une malédiction, mais d'une mauvaise krasis des humeurs de la femme, qui rendait son lait nocif ${ }^{59}$.

Michel Glykas affirme avec force que "c'est pour une raison naturelle que les nouveau-nés sont atteints de maladies et meurent souvent prématurément" ${ }^{60}$. Revenant sur cette question dans son chapitre sur l'aveugle né, il précise que la maladie n'est jamais un châtiment de Dieu, et que c'est la nature qui est à l'origine des maladies des nouveau-nés ${ }^{61}$. De même, l'existence de monstres est imputable à la nature qui façonne l'enfant dans la matrice ${ }^{62}$. C'est la même théorie qu'il avance dans ses Annales, à propos de la naissance d'enfants à deux têtes ou quatre pieds ${ }^{63}$.

$\mathrm{Au} 13^{\mathrm{e}} \mathrm{s}$., Nicéphore Blemmydès cite à l'appui de cette thèse naturaliste Jean Chrysostome, qui avance comme causes à la mort prématurée des nourrissons la mauvaise santé de la mère, la négligence de la nourrice, l'anomalie des airs ou toute autre raison naturelle ${ }^{64}$. La naissance d'enfants mal formés s'explique de même, comme l'a montré Isidore de Péluse, par une union conjugale pendant les règles de la

56. Diodore de 'Tarse, ap. Photios, Bibliolhèque, cod. 223.

57. Pseudo-Athanase, Hom. sur l'aveugle-né, PG 28, 1005.

58. Anastase le Sinaïte, Erotapokriseis, Qu. 88, PG 89, 715. Cf. qu. 96, PG 89, 736.

59. Ibidem, $Q u .127, P G 89,777$. Cf. qu. $96, P G 89,745$. L'anecdote est reprise au $12^{c}$ s. par Michel Glykas, qui l'enfle en attribuant cinq enfants morts à la femme avant qu'elle ne s'avise de chercher une nourrice pour le sixième.

60. Michel Glykas. A porie 37, éd. Eustratiadès, I, p. 423.

61. Idem, Aporie 51, II, p. 64-65.

62. Ibidem.

63. Michel Glykas. Annales, I, p. 54.

64. Jean Chrysostome, A Stagirios affligé par un démon, I, $8(P(; 47,444)$. — Vicéphore Blemmydès, Dialogue sur les bornes de la vie, c. 7, éd. W. Lackner, Nikephoros Blemmydes. Gegen die Vorherbestimmung der Todesstunde, Athènes/Leiden 1985, p. 12. 
femme, quand le sang de cette dernière est impur ${ }^{65}$. Cet amalgame entre une explication rationnelle et le tabou de l'impureté des règles cache une réflexion déculpabilisante : c'est parce que le sang des règles est de mauvaise qualité, et non parce que le couple a transgressé un tabou, que l'enfant est mal formé ${ }^{66}$.

Ces interprétations "naturalistes" provoqueront vers la fin de l'empire une réaction de la part d'auteurs plus soucieux de la toutepuissance du créateur que de l'autonomie des causes naturelles ${ }^{67}$. Il n'empêche que toute cette réflexion a contribué, du moins dans les milieux intellectuels et religieux, à promouvoir une approche plus sereine de la naissance, de la mortinatalité, des enfants malformés : point de magie, point de démons de la naissance ni de Gillo dévoreuse de nouveau-nés, point de funestes présages ou de colère divine, mais le fonctionnement parfois imparfait de la nature créée.

\section{Protéger le nouveau-né}

Ce nouveau-né qui fait moins peur, qui est l'image de Dieu et appelé au salut, devient un être humain dont la vie doit être respectée et protégée par la loi.

Des débuts du christianisme datent les premières lois contre l'infanticide, aussi bien dans la législation impériale que dans les canons.

Aux lois punissant l'infanticide avéré par suffocation ou refus de nourrir, s'ajoutent la répression de l'abandon et de l'exposition (infanticides à peine déguisés), ainsi que l'interdiction de réduire en esclavage les enfants trouvés ${ }^{68}$.

Le droit canon s'attaque lui aussi à l'abandon et à l'exposition, formes les plus répandues de l'infanticide, mais aussi à l'homicide par négligence volontaire ou non (accouchement sur les chemins), aux accidents douteux (enfant trouvé mort étouffé dans le lit de ses parents, accident pour lequel est requis un grand discernement). Dans cette législation canonique, dans ses commentaires et dans les Pénitentiels qui en découlent, la défense de l'enfant se fait rarement au détriment de la mère, pour laquelle on cherche la plupart du temps à discerner d'éventuelles circonstances atténuantes ${ }^{69}$. Dans tous les cas,

65. Ibidem, p. 15. Cif. Isidore de Péluse, Lettre IV, 117, $P G 78,1192$.

66. C'était une opinion commune chez les médecins de la fin de l'Antiquité : cf. Soranos, Maladies des femmes I 12, èd. D. Gourevitch, 1988, p. 33 s. : la période des règles est impropre a la conception parce que "l'ecoulement dilue la semence".

67. Cif. Georgios Scholarios, Sur la providence, èd. Petit-Sidéridès, I, p. 399 ; 432.

68. (.f. par exemple Digeste 25, 3, 4 et Code.Justinien 9, 16, 8, 6 (contre l'infanticide direct) : Novelle 153 de Justinien, Code Justinien 8, 51, 2-3 (contre l'abandon et l'exposition, et l'exploitation des enfants trouvés). Ces différentes lois compilées par Justinien seront reprises dans toute la législation postérieure.

69. Sur l'abandon : canon 15 de Gangres et ses commentaires. Sur l'enfant trouvé mort dans le lit des parent.s : canon 25 de Gangres et ses commentaires, qui vont 
la faute est plus grave si l'enfant n'était pas baptisé, car on le frustre non seulement de la vie terrestre mais encore de la vie éternelle.

3. Bon sens et charité se donnent la main

En instaurant une relation plus sereine au nouveau-né, la christianisation accentue un adoucissement des mours, déjà inauguré par les médecins et philosophes (stoïciens par exemple) de l'époque romaine.

\section{a) Accueillir}

L'effort pour accueillir l'enfant se traduit à la fois dans les institutions (orphanotrophia et brephotrophia) et dans les mentalités : Jean l'Aumônier, durant la grande famine d'Alexandrie, crée des maternités pour les femmes pauvres ${ }^{70}$. De nombreuses Vies de saints présentent des thaumaturges sauvant mère et enfant lors d'accouchements pathologiques ${ }^{71}$.

b) I a charité transgresse les tabous

L'un de ces récits hagiographiques est particulièrement significatif, car le saint n'y opère pas de miracle : il met, si l'on peut dire, la main à la pâte. Un moine d'Ancyre se voit une nuit appelé à la rescousse par un mari dont la femme ne parvient pas à accoucher; délaissant ses prières, le bon moine fait alors office de sage-femme, "sans être rebuté par la souillure inhérente aux femmes qui accouchent, grâce à la charité qui l'y rendait insensible" ${ }^{72}$. Nous voyons dans cet exemple comment la charité transgresse sereinement les tabous.

De même, les exceptions faites à la date du baptême si l'enfant est en péril de mort, le refus d'admettre la damnation des enfants morts sans baptême, la résurrection provisoire en vue de baptiser un enfant mort, et la réponse bourrue de Pierre le chartophylax déclarant que la mère non purifiée doit allaiter son enfant baptisé, relèvent de cet effort pour promouvoir un regard plus positif et des pratiques plus humaines dans le domaine de la naissance.

progressivement dans le sens d'une plus grande prudence avant de conclure a un infanticide volontaire : cf. le Kanonikon de Jean le Jeùneur, repris dans la lettre du chartophylax Vicephore à 'Theodose de Corinthe (HP' V. p. 400).

70. Vie de .Jean l'Aumonier. c. 7. éd. H. Delehaye, An. Boll. 45, 1927, p. 22.

71. Vies de Mélanie, de Porphyre de Gaza, de Syméon Salos, de Marcel l'Acémète. d'Ignace de Constantinople. de Théphano. Histoire Lausiaque et Histoire Philothé. . 1 .

7.2. Histoire Lamsiaque. ( . is. 


\section{Conclusion}

Très progressivement, en charriant de nombreuses survivances de la conception magique du monde, en affrontant de nombreuses résistances, l'Église byzantine a inoculé son anthropologie spécifique dans les mentalités et pratiques concernant le nouveau-né. Celui-ci est peu à peu considéré comme un être humain, et non plus comme cette énigme fragile et menaçante, lieu de toutes les terreurs. La peur de voir naître un enfant non viable ou mal formé demeure, mais elle est tout doucement apprivoisée. On peut en voir un signe dans l'attitude de Michel Psellos, qui porte en lui toutes les contradictions byzantines : dans une lettre à un jeune père, il déclare qu'il "bondit, pour ainsi dire, sur les nouveau-nés", et il décrit en des termes très modernes son attendrissement devant les bébés. Par ce texte du $11^{\mathrm{e}} \mathrm{s}$., Byzance inaugure, bien avant le $18^{\mathrm{e}}$ siècle français, ce que les historiens appellent le "sentiment de l'enfance»" ${ }^{73}$.

Marie-Hélène Congourdeau

C.N.R.S.-URA 186

73. Lettre 157 à l'épi tôn kriséôn, éd. Sathas, V, p. 409. 